\title{
Eigeneinrichtungen innerhalb der GKV - Eine Bewertung aus ökonomischer Sicht
}

Die Gründe für den Ausgabenanstieg im deutschen Gesundheitswesen sind vielfältig und werden unter anderem mit der demographischen Entwicklung und dem medizinisch-technischen Fortschritt erklärt. Der Ausgabenanstieg ist dann nicht negativ zu bewerten, wenn geänderte Präferenzen der Gesellschaft zu einem Ansteigen der Gesundheitsausgaben führen. Negativ zu bewerten ist jedoch, wenn der Kostenanstieg auf Ineffizienzen innerhalb des Gesundheitssystems zurückzuführen ist. Sie sind vor allem Folge von Fehlanreizen auf Seiten der Leistungserstellung sowie organisatorische Mängel in der Versorgung und regulatorischen Handlungsbeschränkungen, die dazu führen, dass Maßnahmen zur besseren Nutzung der Mittel im Gesundheitswesen ergriffen werden. Angesichts aktueller Finanzierungsprobleme in der gesetzlichen Krankenversicherung (GKV) sowie struktureller Probleme bei der Organisation der Gesundheitsversorgung ist es daher notwendig, nach geeigneten Handlungsalternativen zu suchen.

\section{Thomas Plagemann, Sebastian Braun, J.-Matthias Graf von der Schulenburg}

\section{Das Konzept „Eigeneinrichtung“}

Im Gesundheitswesen der USA sind die so genannten „Health Maintenance Organizations“ (HMO) seit mehreren Jahrzehnten ein wesentlicher Bestandteil der Versorgung. Die Zusammenführung der Versicherungs- und Leistungsfunktion in einer Organisation ist in Deutschland grundsätzlich verboten. Ziel der hier vorgestellten repräsentativen Befragung ist die Darstellung eines aktuellen Meinungsbildes innerhalb der GKV zu dem Verbot von Eigeneinrichtungen.

Durch die Zusammenführung der Versicherungs- und Leistungsfunktion in einer Organisation, der so genannten „Health Maintenance Organization“ (HMO), nutzen die USA bereits seit mehreren Jahrzehnten ein mehrere Wertschöpfungsstufen vertikal integrierendes Konzept, um den Ausgabenanstieg ihres Gesundheitssystems zu kontrollieren. ${ }^{1}$ Obwohl die Vorteile einer vertikalen Integration in Form kasseneigener Versorgungseinrichtungen, wie z. B. angestellte Ärzte oder eigene Krankenhäuser, seit Jahr-

Thomas Plagemann, Leibniz Universität Hannover, Forschungsstelle für Gesundheitsökonomie, Hannover und Herescon GmbH - health economic research \& consulting, Hannover Sebastian Braun und Prof. Dr. J.-Matthias Graf von der Schulenburg, beide Leibniz Universität Hannover, Forschungsstelle für Gesundheitsökonomie, Hannover zehnten bekannt sind, ist sie in Deutschland grundsätzlich verboten. ${ }^{2}$

Das Verbot von Eigeneinrichtungen innerhalb der gesetzlichen Krankenversicherung ist ein vielschichtiges Thema, das sowohl (gesundheits-) ökonomische und rechtliche als auch internationale gesundheitspolitische Fragen aufwirft. Der Ausdruck „Eigeneinrichtungen gesetzlicher Krankenversicherungen " ist in der Literatur nicht eindeutig definiert. Grundsätzlich können unter Einrichtungen im gesundheitswissenschaftlichen Kontext die gemäß dem fünften Sozialgesetzbuch (SGB V) definierten Leistungserbringer verstanden werden. Das sind diejenigen Personengruppen, die Leistungen für die Versicherten der Krankenkassen erbringen, also neben Vertragsärzten unterschiedlicher Fachrichtungen und Psychotherapeuten auch Krankenhäuser, Vorsorge- und Rehabilitationseinrichtungen sowie Heil- und Hilfsmittelerbringer. ${ }^{3}$

Mit dem 1988 im Rahmen des Gesundheitsreformgesetzes eingeführten § 140 SGB V wurde die Möglichkeit der Krankenkassen, Eigeneinrichtungen zu betreiben, dahingehend gesetzlich geregelt, dass nur diejenigen Institutionen weiterbetrieben werden durften, die bereits vor dem 01. Januar 1989, d. h. vor Inkrafttreten des GRG, bestanden. ${ }^{4}$ Mit Erlass des zweiten Absatzes wurden die Regelungen des § 140 SGB V jedoch aufgelockert. Dieser lässt die Möglichkeit zu, neue Einrichtungen zu gründen, wenn Krankenkassen ohne die Beauftragung externer 
Leistungserbringer die Durchführung ihrer Aufgaben nicht sicherstellen können. ${ }^{5}$

Der Gesetzgeber hat mit der Einschränkung des Betriebs von Eigeneinrichtungen durch den § 140 SGB V nicht die Beschränkung einer unter Umständen besonders wirtschaftlichen Versorgungsform bezweckt, sondern durch den Erhalt der strikt gegliederten Versorgungsstruktur die Leistungsfähigkeit des Gesundheitssystems zu stärken versucht. Daneben sollte der $§ 140$ SGB V den so genannten „klassischen Kampf“ der Krankenkassen gegen die Leistungserbringer abwenden. ${ }^{6}$

Welche Gründe aus ökonomischer Sicht für bzw. gegen diese Untersagung sprechen, soll Inhalt dieser Abhandlung sein. Das primäre Ziel ist die Darstellung eines aktuellen Meinungsbildes innerhalb der GKV zum Verbot von Eigeneinrichtungen, anhand der Ergebnisse einer Befragung von Vertretern gesetzlicher Krankenkassen. Im Rahmen einer Vollerhebung unter allen gesetzlichen Krankenkassen sollen die folgenden Fragen beantwortet werden:

1. Wie ist die allgemeine Einschätzung der Krankenkassen zu der ökonomischen Wirksamkeit von Eigeneinrichtungen? Besteht ein generelles Interesse, selbst Leistungen anzubieten, und wurden bereits Erfahrungen im Bereich eigener Einrichtungen gemacht?

2. Wie unterscheiden sich diese Ergebnisse im Bezug auf die Kassengröße, d. h. auf die jeweilige Versichertenanzahl?

3. Können Unterschiede hinsichtlich der räumlichen Ausgestaltung des Versicherungsgebietes der Krankenkassen festgestellt werden?

4. Gibt es in Bezug auf die verschiedenen Kassenarten Abweichungen von den Ergebnissen der Grundgesamtheit?

\section{Methodischer Ansatz der Befragung}

Im Vorfeld der Umfrage wurden alle 192 gesetzlichen Krankenkassen (Stand April 2009) telefonisch kontaktiert, um Ansprechpartner der Funktionsbereiche Unternehmens- und Strategieentwicklung bzw. strategisches Controlling zu ermitteln. Die Motivation zu dieser Vorbereitung war die Annahme, dass die Rücklaufquote der Befragung deutlich höher sei, wenn die auszufüllenden Unterlagen ohne Umwege über weitere Schnittstellen zu den entsprechenden Fachleuten gelangen. Anschließend wurden die untersuchungsrelevanten Daten anhand eines Fragebogens erhoben, für dessen Beantwortung zunächst ein Zeitraum von drei Wochen gegeben wurde. Um die Rücklaufquote zu erhöhen, wurde den Ansprechpartnern bei Nichtbeantwortung ein Erinnerungsschreiben zugesandt. Die Befragung wurde mit SPSS 16.0 ausgewertet.

Die Erhebung wurde als quantitative empirische Befragung mit Hilfe eines selbst entwickelten Fragebogens durchgeführt. Zum einen wurden allgemeine statistische Angaben, wie Krankenkassenart, regionale Verteilung so- wie Anzahl der Mitglieder und Versicherten ermittelt. Zum anderen wurden anhand einer fünfstufigen Likert-Skala die Einstellungen der Krankenkassen zur ökonomischen Wirksamkeit von Eigeneinrichtungen erhoben. Diese wurden thematisch wie folgt unterteilt: „Potentiale der Zusammenführung von Versicherungs- und Leistungsfunktion“ und „Hinderungsgründe für die Errichtung eigener Einrichtungen“.

Zur besseren Einordnung der Antworten wurden zwei weitere Fragestellungen ergänzet: Erstens wurde gefragt, ob die Krankenkassen es sich vorstellen könnten, selbst Leistungen anzubieten. Zweitens wurde erhoben, ob sie bereits Erfahrungen im Bereich der Eigeneinrichtungen sammeln konnten. Der Fragebogen enthielt geschlossene wie offene Fragen.

\section{Ergebnisauswertung}

Von den 192 angeschriebenen Krankenkassen haben nach der ersten Aufforderung 42 und nach Erhalt des Erinnerungsschreibens weitere 37 einen ausgefüllten Fragebogen zurückgesendet. Von den 79 Fragebögen waren 76 vollständig ausgefüllt (Quote: 39,5\%). 38,2\% der Kassen, die geantwortet haben, sind im gesamten Bundesgebiet tätig, eine geringe Mehrheit (42,1\%) ist dagegen regional beschränkt und lediglich 19,7\% der Krankenkassen sind auf ein Unternehmen bzw. eine Branche begrenzt. Die Hälfte hat eine Mitgliederanzahl von unter 100.000, insgesamt repräsentieren die Krankenkassen, die an der Studie teilgenommen haben, etwa 32,5 Millionen Mitglieder, was bei insgesamt rund 70 Millionen Versicherten in der GKV einem Anteil von über $46 \%$ entspricht. Folglich ist die Umfrage in der Lage, die gesetzliche Krankenversicherung in Deutschland zu repräsentieren.

Die Befragten wurden gebeten, ihre individuelle Einschätzung zu insgesamt 15 Aussagen abzugeben, in denen sowohl positive als auch negative mögliche Effekte von Eigeneinrichtungen genannt wurden. Die Untersuchungsergebnisse zeigen, dass Aussagen zu positiven Effekten von Eigeneinrichtungen überwiegend Zustimmung finden (Abbildung 1).

Erkennbar ist, dass die Möglichkeiten der Krankenkassen, sich von anderen Kassen im Wettbewerb zu differenzieren sowie Schnittstellen durch eine einheitliche Koordination von Finanzierung und Leistung zu reduzieren, besonders hoch bewertet werden. Daneben wird auch die Wirksamkeit von Eigeneinrichtungen im Hinblick auf die Stärkung der Wirtschaftlichkeit und Wettbewerbsfähigkeit der Krankenkassen sowie eine höhere Unabhängigkeit gegenüber externen Leistungserbringern überwiegend positiv beurteilt. Weniger wirksam dagegen schätzten die Befragten die Funktion von Eigeneinrichtungen zur Verbesserung der Leistungsqualität und der Ressourcenverteilung im Gesundheitswesen ein. Dieses Ergebnis lässt vermuten, dass die Mehrzahl der Krankenkassen der Meinung ist, dass Eigeneinrichtungen vorwiegend der Verbesserung der unternehmensinternen Tätigkeiten und der Wirt- 
Abb. 1: Potentiale von Eigeneinrichtungen

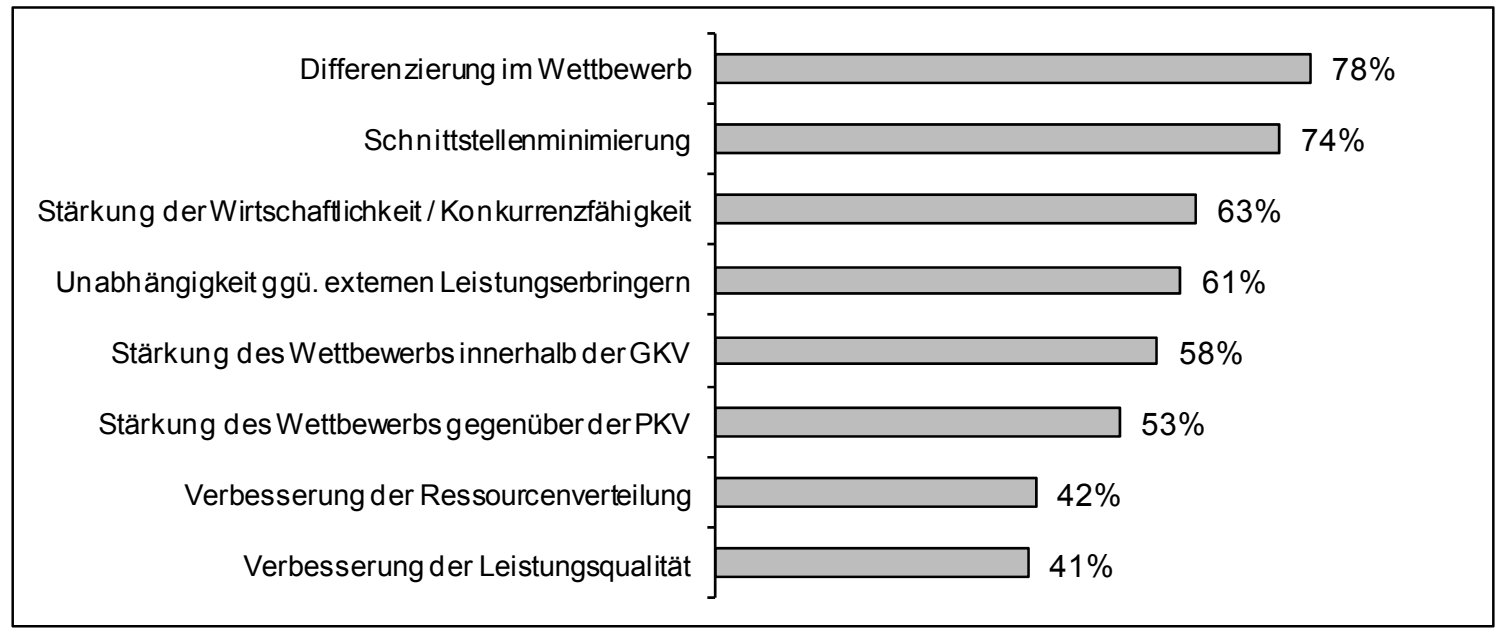

\section{Abb. 2: Bedenken gegenüber Eigeneinrichtungen}

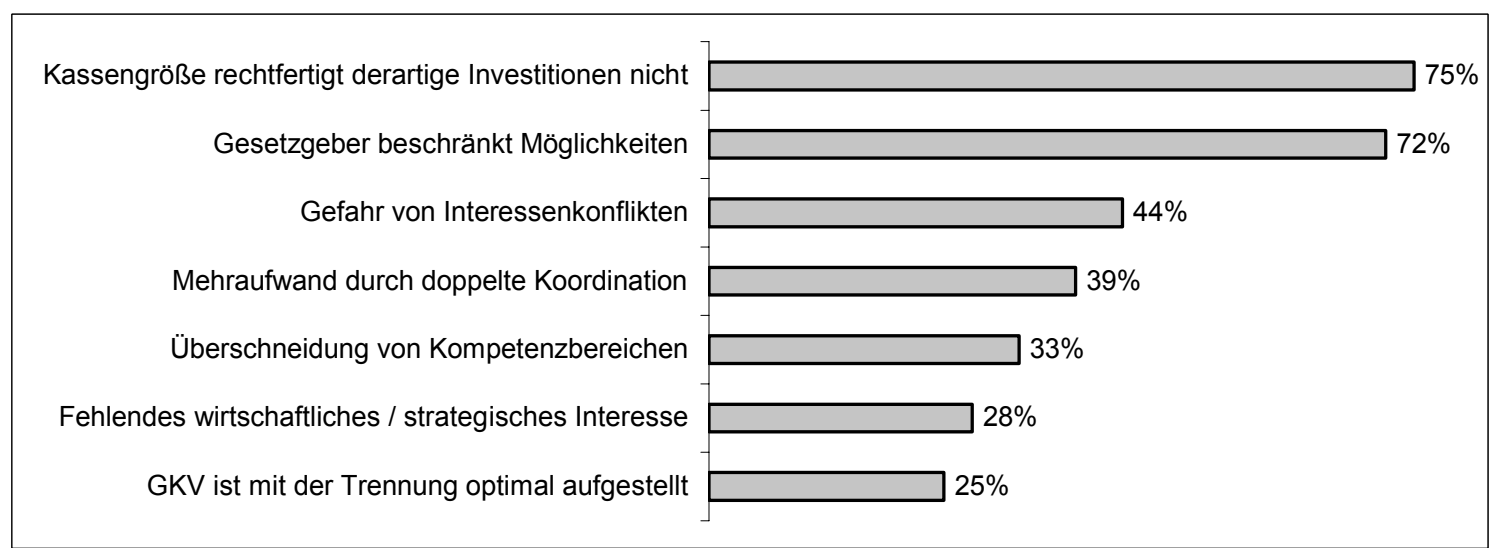

schaftlichkeit von Krankenkassen dienen, Vorteile für die Versicherten dagegen eher von geringerer Bedeutung sind.

Neben den positiven Effekten hat die Umfrage auch sieben Aspekte abgefragt, die der Wirksamkeit von Eigeneinrichtungen hinderlich entgegenstehen könnten. Der überwiegende Teil der Befragten ist dabei der Ansicht, dass eine geringe Zahl an Mitgliedern (geringe Kassengröße) die notwendigen Investitionen in derartige Einrichtungen nicht rechtfertigen würde. Das bedeutet im Umkehrschluss, dass sich Eigeneinrichtungen vorwiegend für diejenigen Krankenkassen rentierten, die über eine hohe Mitgliederzahl verfügen und bei denen die Nachfrage nach bestimmten Versorgungsleistungen entsprechend hoch ist. Daneben wurde bemängelt, dass der Staat den gesetzlichen Krankenkassen nicht ausreichend Möglichkeiten zum Betrieb bzw. zur Gründung von Eigeneinrichtungen einräume (Abbildung 2).
Mögliche Schwierigkeiten bei der internen Durchführung und Koordination von Eigenbetrieben bzw. die Gefahr von Interessenkonflikten zwischen Krankenkassen und Leistungserbringern stellen für die befragten Krankenkassen dagegen weniger bedeutende Hindernisse dar. Es zeigt sich zudem, dass die Teilnehmer eine strikte Trennung von Versicherungs- und Leistungsfunktion grundsätzlich befürworten, aus wirtschaftlichen bzw. strategischen Gründen jedoch teilweise an eigenen Einrichtungen interessiert wären. Als weitere Einwände bezüglich des Betreibens von Eigeneinrichtungen wurden die folgenden Anregungen genannt:

- Die Umsetzbarkeit von Eigeneinrichtungen im deutschen Gesundheitswesen ist aufgrund des hohen poltischen Einflusses der Leistungserbringer und ihrer Interessenvertreter (z. B. Ärzte- und Krankenhausverbände) sowie der Existenz von Körperschaften öffentlichen Rechts, die hoheitliche Aufgaben für Leistungserbringer 
Abb. 3: Potentiale von Eigeneinrichtungen (Cluster „Größe“)

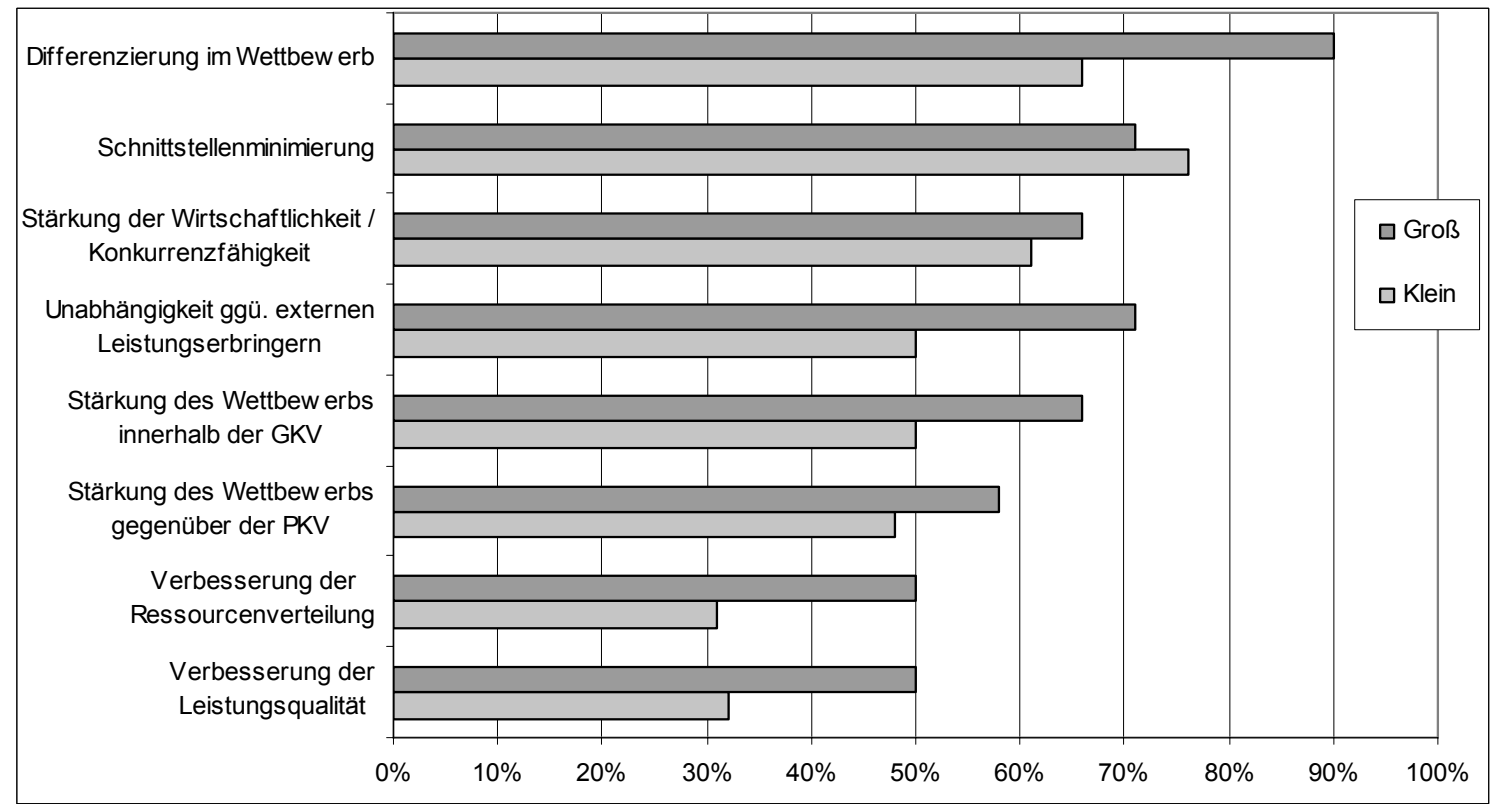

wahrnehmen (z.B. Kassen- und Kassenzahnärztliche Vereinigungen) kaum zu realisieren.

- Politisches Lobbying der Leistungserbringer und die Möglichkeit, die öffentliche Meinung zu beeinflussen, können den Ruf von Krankenkassen, die Eigeneinrichtungen betreiben, schädigen.

- Das Ungleichgewicht im Wettbewerb zwischen den Krankenkassen wird durch die Erlaubnis des Betreibens eigener Einrichtungen verstärkt, sofern diese nicht für alle GKV-Versicherten zur Verfügung stehen.

- Die freie Arztwahl in Deutschland beschränkt die Möglichkeiten, die erzielbaren Effizienzen auszuschöpfen.

Obwohl grundsätzliches Interesse vorliegt, Leistungen selber anzubieten, steht ein Großteil der Krankenkassen Eigeneinrichtungen dennoch kritisch gegenüber. Zum einen stellen sie infrage, ob sich Einrichtungen für alle Kassengrößen rentieren und zum anderen sehen sie erhebliche Probleme bei der Umsetzung im deutschen Gesundheitswesen. Als Begründung wird angegeben, dass der externe Druck (durch die Leistungserbringer, Verbände und die Regierung) die Vorzüge von Eigeneinrichtungen überwiege.

Neben den Einschätzungen der Vor- und Nachteile von Eigeneinrichtungen sollte die Umfrage auch Aufschluss über die Frage geben, wie viele Krankenkassen Interesse an eigenen Einrichtungen haben und ob - trotz des grundsätzlichen Verbots - Erfahrungen mit Eigeneinrichtungen vorliegen. 30,3\% der Befragten bejahten die Frage, ob sie sich vorstellen könnten, eigenständig Leistungen anzubieten, die überwiegende Mehrheit (44,7\%) verneinte sie da- gegen. 11,8\% gaben an, bereits Erfahrungen gesammelt zu haben, vorwiegend gaben die Befragten jedoch an, keine Erfahrungen in diesem Bereich gemacht zu haben (81,6\%), während 6,6\% hierzu keine Aussage machten. Damit wird deutlich, dass die Erfahrungen mit Eigeneinrichtungen innerhalb der GKV in Folge der gesetzlichen Regulierung sehr gering sind.

In einer ersten Subgruppenanalyse wurde das Merkmal „Kassengröße" untersucht. Krankenkassen mit bis zu 100.000 Mitgliedern wurden als „klein“, Kassen mit mehr Mitgliedern als „groß" definiert, so dass beide Gruppen jeweils $50 \%$ der Grundgesamtheit ausmachen. Abbildung 3 illustriert die Ergebnisse bezüglich der Zustimmung bei potentiellen Vorteilen in Abhängigkeit von der Größe:

Die Grafik zeigt deutliche Unterschiede zwischen den Antworten großer und kleiner Krankenkassen. Besonders hohe Abweichungen lassen sich innerhalb der Befragungsitems „Differenzierung im Wettbewerb“, „Unabhängigkeit gegenüber externen Leistungserbringern“ sowie „Stärkung des Wettbewerbs innerhalb der GKV“ identifizieren. Die Abweichung bei den Antworten deutet darauf hin, dass Kassen mit geringer Mitgliederzahl die Wirkung von Eigeneinrichtungen generell schlechter beurteilen als größere. Genau gegenteilige Ergebnisse liefert die Auswertung der Bedenken gegenüber Eigeneinrichtungen: Diese weichen innerhalb des Größen-Clusters ähnlich stark voneinander ab, kleine Krankenkassen gewichten in diesem Fall die Hinderungsgründe jedoch verhältnismäßig höher als große.

Lediglich 15,8\% der kleineren, aber 44,7\% der großen Krankenkassen äußern Interesse an einer eigenen Leistungserstellung. Zusätzlich gaben große Krankenkassen 
Abb. 4: Potentiale von Eigeneinrichtungen (Cluster „Kassenart“")

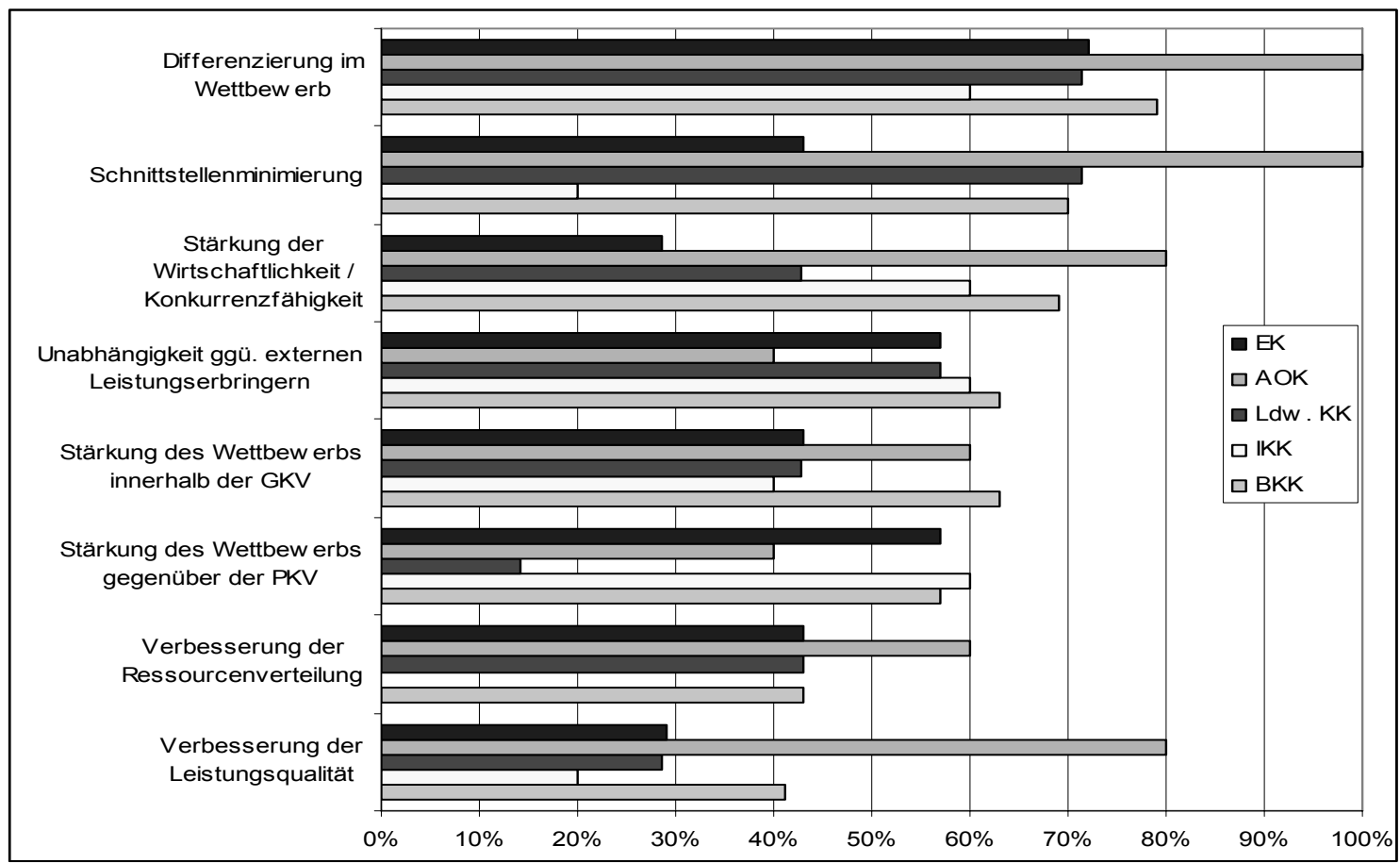

im Vergleich zu kleineren fast viermal so oft an, Erfahrungen mit Eigenbetrieben zu haben, was erneut die These bestätigt, dass die Versicherungsgröße starken Einfluss auf das Engagement der Kassen bezüglich Eigeneinrichtungen haben könnte.

Eine mögliche Ursache für die unterschiedlichen Einschätzungen können Skaleneffekte sein, die mit steigender Größe zunehmen und die Krankenkasse wirtschaftlicher agieren lassen. Auch eine günstigere Risikostruktur der Versicherten ist durch eine hohe Mitgliederzahl zu erreichen, da sich das Krankheitsrisiko tendenziell auf einen größeren Versichertenkreis verteilt. Positiv wirkt sich die Größe auch auf die Liquidität einer Krankenkasse aus, die unverzichtbar im Hinblick auf die Investitionen in kasseneigene Einrichtungen ist. Das mit der Investition verbundene finanzielle Risiko derartiger Projekte ist damit für große Kassen vertretbarer als für kleinere.

Abbildung 4 zeigt die Angaben aufgeteilt nach der Kassenart. Die Betriebskrankenkassen spiegeln dabei in etwa die Angaben der Grundgesamtheit wider. Ersatzkassen bewerten die Sinnhaftigkeit von Eigeneinrichtungen geringer als der Durchschnitt, wohingegen die Allgemeinen Ortskrankenkassen (AOK) in einigen Befragungsitems deutlich positivere Einschätzungen abgaben. Auch im Hinblick auf Erfahrungen mit Eigeneinrichtungen sind die regional aufgestellten AOKen nach eigenen Angaben überdurchschnittlich präsent.

\section{Diskussion}

Das Konzept versicherungseigener Einrichtungen in Form der HMOs stammt aus den USA. Dort gab es diese bereits Anfang des 20. Jahrhunderts in der Ausgestaltung, dass zumeist Unternehmer eigene Organisationen gründeten, in denen sie Ärzte anstellten, um die medizinische Versorgung ihrer Angestellten zu sichern. Dem klassischen amerikanischen HMO-Model liegt die Idee zugrunde, den Leistungserbringern ein festes Budget für die gesamte Krankenversorgung aller Angestellten eines Betriebs zur Verfügung zu stellen, wodurch den Leistungserbringern das Krankheitsrisiko übertragen wird. In den USA gelten HMOs allgemein als probates Mittel, die medizinischen Ausgaben zu verringern. ${ }^{7}$

Auch im deutschen Gesundheitssystem könnten kasseneigene Einrichtungen dazu beitragen, die Prozessabläufe effizienter zu gestalten und Kosten einzusparen. Ebenfalls wirksam könnten Eigeneinrichtungen zur Verbesserung der Beziehungen zwischen den Krankenkassen und ihren Versicherten bzw. zur Gewinnung neuer Mitglieder sein. Aus ökonomischer Sicht kann es daher durchaus sinnvoll sein, das Verbot von Eigeneinrichtungen aufzuheben, da es eine potentiell wirtschaftlichere Versorgungsform untersagt.

Neben positiven Effekten von Eigeneinrichtungen sind jedoch auch eine Vielzahl von negativen Aspekten zu nennen, die das Verbot kasseneigener Einrichtungen rechtfertigen lassen. So liegen möglicherweise die Vorteile 
der vertikalen Integration im Gesundheitswesen vorwiegend bei den Krankenkassen. Diese wären in der Lage, ihre Ergebnissituation durch Eigeneinrichtungen und die direkte Anstellung eigener Ärzte zu verbessern und darüber Kosten einzusparen. Diese Vorteile können die Krankenkassen jedoch nur generieren, indem sie die Entscheidungsfreiheiten anderer Beteiligter des Gesundheitserstellungsprozesses einschränken. Kürzung des Einkommens und Einschränkung der unternehmerischen und therapeutischen Freiheit der Leistungserbringer können - so die Argumentation - zu Mängeln in der Versorgungsqualität führen. Im schlimmsten Fall wäre es sogar denkbar, dass Ärzte bzw. die dahinter stehenden Krankenkassen Leistung gänzlich verweigern. Entsprechende gesundheitspolitische Maßnahmen, um diese Leistungsmängel auszuschließen, erfordern einen erhöhten Kontrollaufwand, der wiederum zu einem Anstieg der Ausgaben führen und die erzielbaren Vorteile beseitigen würde.

Der Betrieb kasseneigener Eigeneinrichtung würde zudem erfordern, dass Versicherte verpflichtet werden könnten, Behandlungsleistungen in einer vorgeschriebenen Institution nachzufragen, wie es bspw. im amerikanischen HMO-System der Fall ist. In den USA sind die HMOs derzeit die dominierende Form der Krankenversicherung. ${ }^{8}$ Auch die mit einer HMO-Mitgliedschaft verbundene Einschränkung der freien Arztwahl konnte dieser Entwicklung nicht schaden: 1990 wurden etwa 90\% der Behandlungen von HMO-Versicherten innerhalb des HMO-Systems durchgeführt, in nur etwa $10 \%$ der Fälle wurden diese von externen Leistungserbringern behandelt. ${ }^{9}$ Diese Akzeptanz der Versicherten ist vermutlich auf die Tatsache zurückzuführen, dass das Versicherungssystem der USA bis Mitte der dreißiger Jahre primär aus privaten Krankenversicherungen einerseits und einer kostenintensiven staatlichen Gesundheitsversorgung andererseits bestand. Eine allgemeine Krankenversicherung mit freier Arztwahl, wie sie etwa in Deutschland seit Ende des 19. Jahrhunderts existiert, konnte sich in den USA dagegen bis heute nicht etablieren, sodass HMOs trotz einiger Einschränkungen einen großen Fortschritt in dem amerikanischen Gesundheitssystem bedeuten. ${ }^{10}$

Für das deutsche Gesundheitssystem ist eine Einschränkung der freien Arztwahl zumindest zum heutigen Zeitpunkt nicht vorstellbar. Erste Erfahrungen mit den Selektivverträgen zur hausarztzentrierten und integrierten Versorgung verdeutlichen diese Problematik. Der Grund dafür liegt in dem in Deutschland gepflegten Paradigma der Wahlfreiheit des Patienten. Es ist jedoch nicht auszuschließen, dass eben diese Freiheiten im Hinblick auf die Ausgabenentwicklung vom Gesetzgeber zukünftig eingeschränkt werden.

Doch selbst in den USA stehen mehr und mehr Versicherte genau wegen der Einschränkung der Patientenrechte, wie z. B. der freien Arztwahl, den HMOs kritisch gegenüber. ${ }^{11}$ Die aktuellen Entwicklungen der amerikanischen HMOs verlaufen dahingehend, dass die Versicherten zu denjenigen HMOs abwandern, die ihnen mehr Entscheidungsspielräume, wie z. B. die freie Arztwahl oder aber Kostenersparnisse durch Prämienrückerstattungen, ermöglichen. ${ }^{12}$ Zurzeit sind in den USA knapp 70 Millionen Bürger in einer HMO versichert, was etwa $23 \%$ der amerikanischen Bevölkerung ausmacht. ${ }^{13}$

Damit kasseneigene Einrichtungen unter gesamtwirtschaftlichen und gesundheitsökonomischen Gesichtspunkten Nutzen erzeugen können, bedarf es weiterer Voraussetzungen. Ein Grund, warum kasseneigene Betriebe in Deutschland derart wenig vertreten sind, wie die Befragung ergeben hat, liegt sicher nicht nur in dem Verbot von Eigeneinrichtungen sondern auch in einer derzeit noch bestehenden schlechten Kosten-Nutzen-Relation. Hält der Trend der vergangenen Jahre von Fusionen unter den Krankenkassen an, so könnten sich Eigeneinrichtungen als wirksames Instrument zur Kostenreduzierung entwickeln. Durch eine gestiegene Versichertenanzahl pro Krankenkasse und die dadurch entstehenden finanziellen Möglichkeiten hätten die verbleibenden Krankenkassen eher Anreize und Chancen, auch über Eigeneinrichtungen miteinander in Wettbewerb zu treten.

Es stellt sich die Frage, ob Kosteneinsparungen durch Eigeneinrichtungen das Gesundheitswesen finanziell entlasten oder die Kostenvorteile alleinig von den Krankenkassen einbehalten werden. Für die Außenwirkung einer einzelnen Krankenkasse ist keinesfalls gesagt, dass die (nun einmal explizit angenommene) Optimierung der Leistungserstellung nur Vorteile hat. Krankenkassen, die durch den Betrieb eigener Einrichtungen Vorteil erwirtschaften, könnten in der Öffentlichkeit auch den Eindruck erwecken, eher an der Gesundheit ihrer Versicherten zu sparen, als dass die Kostenreduzierungen auf eine Optimierung der Abläufe bzw. eine Verringerung von Schnittstellen zurück zu führen sind.

\section{Schlussfolgerung}

Trotz der potentiellen ökonomischen Vorteile einer vertikalen Integration von Versicherung und Leistungserbringung im deutschen Gesundheitswesen, werden kasseneigene Einrichtungen nach dem Vorbild der amerikanischen HMOs in Deutschland zumindest in absehbarer Zukunft Seltenheitswert behalten. Auch ist das deutsche Gesundheitssystem in seiner generellen Ausrichtung für Eigeneinrichtungen von Krankenkassen noch nicht vorbereitet. Ein breites Interesse die Sinnhaftigkeit des grundsätzlichen Verbots von Eigeneinrichtungen in den Fokus gesundheitspolitischer Diskussionen zu stellen, besteht von Seiten der Krankenkassen nicht.

\section{Literatur}

Altman, S. H. / Wallack, S. (1996): Health care spending: Can the United States control it?, in: Altman, S. H. / Reinhardt, U. E. (Hrsg.), Strategic choices for a changing health care system, Chicago.

Amelung, V. E. / Schumacher, H. (2001): Patient Relationship Management CRM in der Life Sciences Industrie, Wiesbaden.

Beck, T. F. (2008): Managed Care in der stationären Leistungserbringung: Innovative Integrierte Versorgung als Chance für Krankenhäuser, Hamburg. 


\section{THEMA}

Franken, A. (2003): Die privatrechtliche Binnenstruktur der integrierten Versorgung, §§ 140 a-h SGB V, in: Juristische Reihe Tenea (Hrsg.), Bd. 29 , Berlin.

Mühlbacher, A. (2002): Integrierte Versorgung: Management und Organisation: Eine wirtschaftswissenschaftliche Analyse von Unternehmensnetzwerken der Gesundheitsversorgung, Bern, Göttingen, Torronto.

Orlowski, U. / Rau, F. / Schermer, J. (Hrsg.) (2007): SGB V-Kommentar - Gesetzliche Krankenversicherung, Heidelberg, München, Berlin.

Poorsina, R. (2009): About Kaiser Permanente, URL: http://xnet.kp.org/ newscenter/pressreleases/ nat/2009/042209myhealthmgr.html [Stand 05.05.2009].

Quaas, M. / Zuck, R. (2004): Medizinrecht in: Schriftenreihe der Neuen Juristischen Wochenschrift, Bd. 72, München.

Russell, H. M. (2004): HMO-PPO/Medicare/Medicaid Digest, Managed Care Digest Series, Kansas.

Schulenburg, J.-M. Graf von der (1982): Health Maintenance Organizations Eine interessante Alternative zur traditionellen Krankenversicherung?, Zeitschrift für die gesamte Versicherungswissenschaft, Jg. 71 (1982), Berlin Heidelberg. S. 627-648.
Wagner, E. (1997): Types of Managed Care Organizations, in: Kongstvedt, P. R. (Hrsg.), Essentials of Managed Health Care, 2. Aufl., Gaithersburg. Wiechmann, M. (2003): Managed Care - Grundlagen, internationale Erfahrungen und Umsetzung im deutschen Gesundheitssystem, Wiesbaden.

\section{Fußnoten}

1 Vgl. Beck, T. F. (2008), S. 6; Altman, S. H. / Wallack, S. (1996), S. 272-283.

2 Vgl. Schulenburg, J.-M. Graf von der (1982), S. 627-648.

3 Vgl. Quaas, M. / Zuck, R. (2004), S. 159-162, Rn. 6.

4 Vgl. Franken, A. (2003), S.139.

5 Vgl. $\$ 140$ Abs. 2 S. 1 SGB V.

6 Vgl. Orlowski, U. / Rau, F. / Schermer, J. (2007), S. 108.

7 Vgl. Poorsina, R. (2009)

8 Vgl. Poorsina, R. (2009).

9 Vgl. Wiechmann (2003), S. 55; Wagner, E. (1997), S. 41.

10 Vgl. Mühlbacher, A. (2002), S. 40.

11 Vgl. Amelung, V. E. / Schumacher, H. (2001), S. 52.

12 Vgl. Russell, H. M. (2004), S. 176.

13 Vgl. Wiechmann, M. (2003), S. 77.

\section{Gesundheitssport}

\section{Ein vernachlässigtes Mittel zur Förderung der öffentlichen Gesundheit}

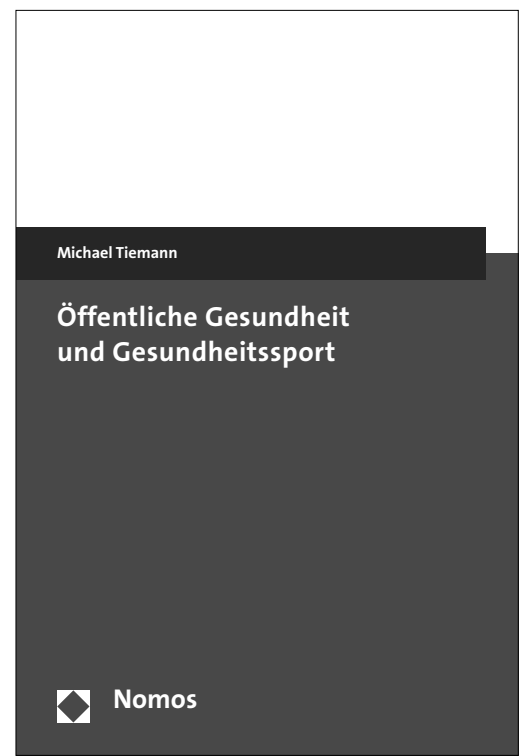

\section{Öffentliche Gesundheit und Gesundheitssport}

Von PD Dr. Michael Tiemann

2010, 281 S., brosch., 49,90€, ISBN 978-3-8329-5696-7

Bitte bestellen Sie im Buchhandel oder versandkostenfrei unter - www.nomos-shop.de
Körperlich-sportliche Aktivität und spezifische Gesundheitssportprogramme beinhalten ein großes Potenzial zur Förderung der Öffentlichen Gesundheit. Dieses wird bislang allerdings wenig genutzt, wobei insbesondere die fehlende Vernetzung zwischen Akteuren des Sport- und des Gesundheitssystems ein großes Manko darstellt.

Der Band gibt einen detaillierten Überblick über bedeutsame Ansätze zur Förderung der Öffentlichen Gesundheit durch körperlich-sportliche Aktivität und deren Evidenzbasierung. Weiter wird ein neues, über einen 7-jährigen Zeitraum erfolgreich erprobtes Konzept zur Vernetzung von Ärzten, Krankenkassen und Gesundheitssportanbietern vorgestellt. Besonders bemerkenswert ist dabei die Beteiligung von über 1.300 Ärzten. Des Weiteren werden die Effekte der in diesem Kontext eingesetzten Gesundheitssportprogramme für inaktive und gesundheitlich belastete Erwachsene dargestellt. Neben Verbesserungen des Gesundheitsstatus und Gesundheitsverhaltens konnten damit auch positive Veränderungen im Hinblick auf Gesundheitskosten erzielt werden.

Die Arbeit richtet sich vor allem an Gesundheits- und Sportwissenschaftler, Ärzte und weitere Gesundheitsexperten, z.B. von Krankenkassen. 